\title{
AN ACCELERATION METHOD FOR NUMERICAL STUDIES OF CONJUGATE HEAT TRANSFER WITH A SELF-ADAPTIVE COUPLING TIME STEP METHOD: APPLICATION TO A WALL-IMPINGING FLAME.
}

\author{
Chai KOREN ${ }^{\ddagger}$, Ronan VICQUELIN $\$$, Olivier GICQUEL ${ }^{\S}$ \\ $\S$ Laboratoire EM2C, CNRS \\ CentraleSupélec \\ Université Paris-Saclay \\ Grande Voie des Vignes, \\ 92295 Chatenay-Malabry cedex, France \\ \#Air Liquide \\ Centre de Recherche Paris-Saclay \\ 1 Chemin de la Porte des Loges, \\ 78350 Les-Loges-en-Josas, France
}

\begin{abstract}
The application of large-eddy simulations to conjugate heat transfer problems can promisingly provide accurate results, including fluctuating heat loads which are critical for thermal fatigue. Such simulations rely on separate solvers and a coupling methodology which must be accurate and robust. In this context, the Hybrid-Cell Neumann-Dirichlet (HCND) coupling approach can adapt dynamically the coupling frequency given a desired accuracy. However, in order to determine statistics (mean, RMS, ...) in a permanent regime, this approach must benefit from an acceleration technique which is here first derived and validated. Two configurations of a wall-impinging flame are then simulated: a quasi-steady case and a pulsated case. The former enables to validate the ability of the accelerated HCND method to predict a steady state wall temperature, while the latter highlights the retained acceleration which does not alter the fluctuations in wall temperature and wall heat flux. Both cases benefit from the selfadaptation of the coupling period provided by the method.
\end{abstract}

\section{NOMENCLATURE}

$A_{F} \quad$ Flame thickening amplitude [-]

\footnotetext{
*Address all correspondence to this author: ronan.vicquelin@centralesupelec.fr.
}

$c_{p}$ Thermal capacity at constant pressure $[\mathrm{J} / \mathrm{kg} / \mathrm{K}]$

$D$ Diamater $[\mathrm{m}]$

$f \quad$ Frequency [Hz]

$F \quad$ Local thickening factor [-]

$\mathscr{J}_{k} \quad$ Diffusive flux of species $k\left[\mathrm{~kg} / \mathrm{s} / \mathrm{m}^{2}\right]$

$\dot{m}$ Mass flowrate [kg/s]

$S_{F} \quad$ Flame sensor [-]

$t$ Time [s]

$T$ Temperature [K]

$U$ Bulk velocity $[\mathrm{m} / \mathrm{s}]$

$V \quad$ Cell volume $\left[\mathrm{m}^{3}\right]$

$Y_{c} \quad$ Progress variable [-]

$Y_{(\ldots)} \quad$ Species mass fraction [-]

$\alpha$ Desynchronization factor [-]

$\Delta t \quad$ Time step size [s]

$\eta \quad$ Numerical tolerance [-]

$\lambda$ Thermal conductivity $[\mathrm{W} / \mathrm{m} / \mathrm{K}]$

$\dot{\omega}$ Reaction rate $\left[\mathrm{s}^{-1}\right]$

$\Phi$ Face-integrated flux [W]

$\rho$ Density $\left[\mathrm{kg} / \mathrm{m}^{3}\right]$

$\tau_{\text {cond }}$ Characteristic conductive time scale [s]

$(\ldots)_{f}$ Fluid domain value

$(\ldots)_{\text {bnd }}$ Boundary value

$(\ldots)_{c p l}$ Coupling value 
$(\ldots)_{\text {ext }}$ External imposed conditions

$(\ldots)_{s}$ Solid domain value

$(\ldots)_{\text {inj }}$ Injector characteristics

$\overline{(\ldots)}$ Mean value

(...) Numerical estimation of mean value

$(\ldots)^{\prime} \quad$ Fluctuating contribution

CHT Conjugate Heat Transfer

HCND Hybrid-Cell Neumann-Dirichlet

LES Large Eddy Simulation

PID Proportional-Integral-Derivative

RANS Reynolds-Averaged Navier-Stokes

RMS Root Mean Square value

\section{INTRODUCTION}

Wall heat transfer in combustors affects the structure life cycle. Numerical simulations to predict wall heat fluxes and temperature fields can help to determine the best compromise in terms of choice of material and cooling system. The estimation of wall heat loads then requires accurate description of both heat conduction in solid parts and the reactive flow inside the combustor. The study of conjugate heat transfer (CHT), not necessarily involving combustion, is an active area of research. Using code coupling between separate solvers used for the solid part and the fluid flow have been carried out for RANS simulations [1-4] and for Large Eddy Simulations (LES) [5-10] . When studying turbulent reactive flows, the more and more maturing LES framework offers great accuracy [11] compared to RANS, at the cost of consuming large computational resources. The requirement for high-fidelity methods is all the more true in critical conditions such as flames in the close vicinity of walls. The combination of conjugate heat transfer and large-eddy simulations is then a promising candidate for such desired methods.

When coupling codes for conjugate heat transfer problems, a particular attention must be paid to the coupling methodology and the boundary conditions applied to each solvers between iterations. For unsteady CHT studies with flow and solid solvers that are both unsteady, a standard approach is the Neumann-Dirichlet coupling method where, between coupling events, one solver is given a fixed temperature at the interface (Dirichlet condition) while the other one uses a Neumann condition with a given value of the wall heat flux from the first solver. The stability of this approach has been studied [12] and enhanced [6, 8, 13-15].

The term permanent regime is here used to denote the final behavior of the system after an initial transient phase. In a general case, this final state (in turbulent or oscillatory flows) remains unsteady and is characterized by a spectrum of harmonics. Steady conditions here denote conditions that do not present any temporal variations. An issue to deal with in unsteady simulations of CHT problems is the slow conduction process such that the transient heating or cooling of a solid part to its permanent state requires to simulate a physical time which is not affordable. This is alleviated by artificially accelerating the physical transient to reach sooner the permanent regime which is still unsteady in a pulsated or turbulent flow. Several techniques have been proposed in the literature to carry out such an acceleration $[5,9,13,14,16,17]$. A first example is the coupling of an unsteady flow solver with a steady solid heat transfer solver [16]. By doing so, the mean fields are obtained at a low computational cost but this approach is limited to steady-state computations and cannot grant access to the temperature and heat flux fluctuations in the wall. A second approach is the desynchronization method $[5,9,17]$ which can be used with fully unsteady coupled simulations. In this method, codes are no longer synchronized in terms of physical time: while the flow solver simulates a physical time of $\Delta t_{c p l}$ the solid heat transfer solver simulates a physical time of $\alpha \Delta t_{c p l}$ where $\alpha \approx 100-500$. This methodology provides an efficient and robust mean to compute the mean temperature and wall heat flux fields on the interface boundary. However, by modifying the frequencies perceived by the solid, such a technique amplifies the level of heat loads fluctuations. In practice then, once the permanent regime is reached, the acceleration algorithm is stopped.

To be affordable, conjugate heat transfer with LES must be carried out on massively parallel clusters. In these unsteady simulations, both flow and heat conduction solvers exchange data at their interface regularly after a prescribed coupling time step $\Delta t_{c p l}$. The optimal value of the latter quantity is not known. The recently developed Hybrid-Cell NeumannDirichlet (HCND) method [18] is here considered to determine this coupling time step dynamically from an accuracy tolerance. This makes the HCND method relevant to high-fidelity unsteady numerical studies of conjugate heat transfer when considering a direct numerical or large-eddy simulation in the flow field. The objectives of the present study are twofold: i) Adapt the HCND method through an acceleration method to compute conjugate heat transfer statistics in permanent regimes, and ii) apply the enhanced method to a wall-impinging flame configuration and compare it to a standard coupling method. Before addressing more practical turbulent flames, the methodology is first validated in a laminar flame in order to reduce the number of modeling uncertainties. Addressing the considered flame configuration in a $2 \mathrm{D}$ axisymmetric domain is the most efficient approach. However, since our envisioned future applications are 3D coupled LES, we have implemented the derived accelerated HCND method in unsteady 3D solvers directly. While this leads to significantly larger computationally resources for the case investigated in the present study compared to a $2 \mathrm{D}$ simulation, the methodology validated here with the chosen solvers will then enable us to rapidly consider unsteady coupled 3D simulations of practical turbulent flows.

The Hybrid-Cell Neumann-Dirichlet method is first briefly detailed before deriving and validating an acceleration method to 
reach the permanent regime of the simulated system much faster. The studied wall-impinging flame configuration [19] is then described along with the retained physical modeling and numerical setup. Thanks to the acceleration of the method, computing the wall temperature becomes affordable with the HCND method whose results are compared to a standard Neumann-Dirichlet coupling approach accelerated by desynchronization. Finally, in order to induce fluctuations in wall temperature and heat flux, the flame jet mass flowrate is pulsated to check the behavior of the enhanced method in an unsteady conjugate heat transfer problem with flow dynamics.

\section{HYBRID-CELL NEUMANN-DIRICHLET METHOD AND ACCELERATION OF THE TRANSIENT REGIME}

The Hybrid-Cell Neumann-Dirichlet method is first briefly presented before being enhanced to artificially accelerate the transient heating or cooling of the structure. Validations are carried out on a $1 \mathrm{D}$ unsteady $\mathrm{CHT}$ problem.

\section{Hybrid-Cell Neumann-Dirichlet method}

The Hybrid-Cell Neumann-Dirichlet (HCND) method [18] has been derived for target applications which are coupled DNS/LES of unsteady CHT. For the sake of accuracy of such coupled high-fidelity methods, the coupling time step between communications of separate solvers is self-adapted. This is done by considering hybrid cells around the solid-fluid interface (see Fig. 1) in order to derive the following ordinary differential equation for the boundary temperature:

$$
\frac{d T_{b n d}}{d t}=-\frac{\Phi_{f, b n d}+\Phi_{s, b n d}}{V_{f} \rho_{f} c_{p f}+V_{s} \rho_{s} c_{p s}}
$$

The coupling approach is based on a layer of such hybrid cells at the boundary. The integrated heat flux over all internal faces in the solid domain of the hybrid cell is denoted $\Phi_{s, \text { bnd }}$, and $\Phi_{f, b n d}$ in the fluid. Both quantities are provided by the flow and solid solvers. Dirichlet boundary conditions are then provided for the flow and solid heat transfer solvers. Thanks to this formulation, an automatic determination of the coupling time step is achieved by controlling the numerical integration error using a controller. As detailed in [18], given an estimated numerical error from two temporal integration schemes, the controller adapts the coupling time step for the next coupling stage. Since targeted applications are unsteady $3 \mathrm{D}$ simulations, time step rejection is not considered in order to keep the computational cost affordable. Also, undesired variations in the coupling time step due to the coupled dynamics of the controller and the considered equation system solved with variable time steps must be prevented. This is why a robust PID controller [20] is here considered and has been shown to work satisfactorily [18]. This achieved control of the numerical error also ensures the numerical stability of the coupling procedure. The only parameter is then a prescribed tolerance to control the numerical accuracy of the coupling method.

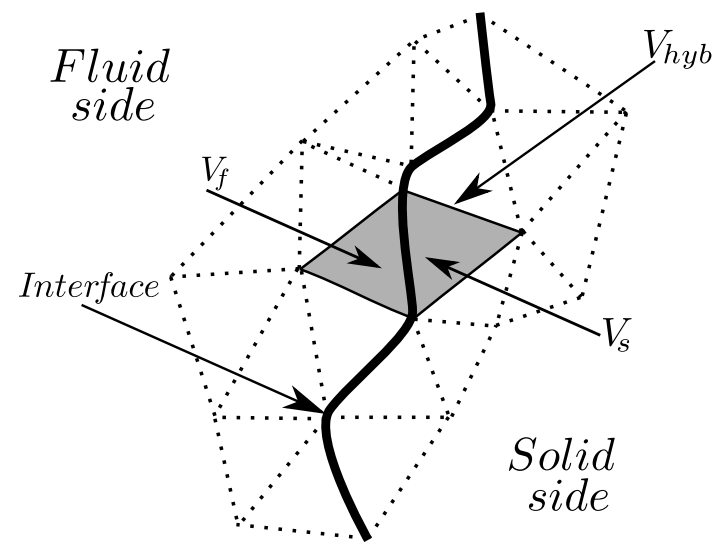

FIGURE 1. One hybrib cell of volume $V_{h y b}$ (in grey) is the union of fluid and solid cells of volume $V_{f}$ and $V_{s}$ located on each side of the interface.

\section{Superposition of mean and fluctuating parts to afford computing permanent regime statistics}

As highlighted in the introduction, the discrepancy between the fluid and solid time scales leads to a huge need in computational resources: computing the transient heating or cooling of a combustion chamber requires simulating a very large physical time. The desynchronization technique is widely used in CHT studies with LES $[5,9,17]$. However, it is not compatible with the HCND coupling method since the boundary temperature is determined by an ordinary differential equation which requires temporal synchronization of heat fluxes from both solvers. For this reason, an acceleration method based on a similar approach to $[13,14]$ is derived: the temperature field inside the walls is described as the superposition of a steady and a transient component: $T_{s}=\widehat{T}_{s}+T_{s}^{\prime}$. Each component is then solved using the adequate heat transfer equation:

$$
\begin{aligned}
0 & =\nabla \cdot\left(\lambda_{s} \nabla \widehat{T}_{s}\right) \\
\rho_{s} c_{p, s} \frac{\partial T_{s}^{\prime}}{\partial t} & =\nabla \cdot\left(\lambda_{s} \nabla T_{s}^{\prime}\right)
\end{aligned}
$$

The instantaneous wall heat flux on the fluid side, $\Phi_{f, b n d}$, is also split into two components: A mean wall heat flux $\widehat{\Phi}_{f, b n d}$ and a 
fluctuating heat flux $\Phi_{f, \text { bnd }}^{\prime}$ such that

$$
\Phi_{f, b n d}^{\prime}=\Phi_{f, b n d}-\widehat{\Phi}_{f, b n d}
$$

Solving Eq. 2 is accomplished by using the mean wall heat flux $\widehat{\Phi}_{f, b n d}$ as a boundary condition on the shared interface, and yields the solid mean temperature field of $\widehat{T}_{s}$ including the interface mean temperature $\widehat{T}_{b n d}$. In the unsteady fluid solver, the estimated mean wall heat flux at a time $\tau$ is computed by time averaging the instantaneous wall heat flux:

$$
\widehat{\Phi}_{f, b n d}(\tau)=\frac{1}{\tau} \int_{0}^{\tau} \Phi_{f, b n d}(t) d t
$$

Once this cumulative sum is long enough, $\widehat{\Phi}_{f, b n d}(\tau)$ converges to the steady mean flux $\bar{\Phi}_{f, b n d}$. The unsteady fluctuating solid temperature equation, Eq. 3, is coupled to a fluctuating variant of the boundary temperature equation, Eq. 1,

$$
\frac{d T_{b n d}^{\prime}}{d t}=-\frac{\Phi_{f, b n d}^{\prime}+\Phi_{s, b n d}^{\prime}}{V_{f} \rho_{f} c_{p f}+V_{s} \rho_{s} c_{p s}}
$$

following the usual HCND coupling method, which still selfadapts the coupling time step given a prescribed tolerance. Finally, the instantaneous boundary temperature, used as a boundary condition by the flow solver, is obtained by summing both parts:

$$
T_{b n d}=\widehat{T}_{b n d}+T_{b n d}^{\prime}
$$

In $[13,14]$, the considered mean/fluctuation splitting is carried out on all retained Fourier modes. The fundamental mode, i.e. the steady state, is solved with a steady heat transfer equation solver while the unsteady heat conduction is solved for other spectral modes in the frequency-domain. Here, keeping a timedomain resolution to describe all types of fluctuations enables to account for a single unsteady equation which is solved numerically with a controlled accuracy. As in $[13,14]$, the acceleration to a permanent regime by disregarding the slow solid conduction process results from providing the mean heat flux $\widehat{\Phi}_{f, b n d}$, which converges within several flow time scales, directly as a boundary condition to the steady conduction problem in Eq. 2. Let us explain this carefully: The superposition principle of a mean state and a fluctuating part is exact as long that the boundary condition applied to Eq. 2 is the exact steady mean flux. During the simulated initial transient, it is not the case since the cumulative average in Eq. 5 (that ultimately reaches a mean steady state) is not converged. Hence, Eq. 2 is forced in a quasi-steady behavior
TABLE 1. Properties of both mediums considered in 1D test cases: Thermal conductivity, density, thermal capacity at constant pressure

\begin{tabular}{cccc}
\hline \multicolumn{2}{c}{ Inconel steel } & \multicolumn{2}{c}{ Burnt gases } \\
\hline$\lambda_{s}$ & $11.70 \mathrm{~W} / \mathrm{m} / \mathrm{K}$ & $\lambda_{f}$ & $0.158 \mathrm{~W} / \mathrm{m} / \mathrm{K}$ \\
$\rho_{s}$ & $8510.0 \mathrm{~kg} / \mathrm{m}^{3}$ & $\rho_{f}$ & $3.65 \mathrm{~kg} / \mathrm{m}^{3}$ \\
$c_{p, s}$ & $439.0 \mathrm{~J} / \mathrm{kg} / \mathrm{K}$ & $c_{p, f}$ & $1738 \mathrm{~J} / \mathrm{kg} / \mathrm{K}$ \\
\hline
\end{tabular}

where the applied non-converged wall heat flux is then instantaneously equilibrated in the solid thermal state. This is how the physical transient is artificially accelerated. Ultimately, the cumulative average in Eq. 5 converges to a steady mean value. This is when the strict validity of the superposition becomes valid and the fluctuations in the reached permanent regime are then not impacted by the acceleration method. Once $\widehat{\Phi}_{f, b n d}$ is converged, Eq. 2 also yields the exact mean steady solution in the solid.

\section{D validation of the accelerated coupling}

The HCND coupling methodology combined with the acceleration method of the thermal transient is tested on the following one-dimensional test case: The test case consists of two coupled one-dimensional codes, each solving the unsteady heat equation in each medium. The two mediums are: The burnt gases of oxycombustion at a 20-bars pressure on the one hand, and an Inconel steel on the other hand. Properties of both mediums are given in table 1. The configuration is shown in Fig. 2: The interface is located at the axial position $x=0$ with the fluid medium on the negative $x$-values and the solid medium at the positive $x$-values. Boundary conditions are applied at both extreme edges of the domain: at $x=-L_{f}$, the fluid temperature fluctuates following a temporal sine wave of frequency $f_{\text {ext }}$,

$$
T_{f, e x t}=T\left(x=-L_{f}, t\right)=T_{0}\left(1+0.1 \sin \left(2 \pi f_{\text {ext }} t\right)\right)
$$

with $T_{0}=1000 \mathrm{~K}$, while at $x=L_{s}$, the solid's outer boundary $T_{s, \text { ext }}$ is a fixed temperature equal to the initial mean temperature in both mediums: $293 \mathrm{~K}$.

The evolution of the resulting interface temperature along time is plotted in Fig. 3. The order of magnitude of the transient time to permanent regime can in fact be estimated by the characteristic conduction time scale $\tau_{\text {cond }}=\delta^{2} / a_{s}$ in the solid, where $\delta$ is a solid characteristic length and $a_{s}=\lambda_{s} /\left(\rho_{s} c_{p_{s}}\right)$ the solid thermal diffusivity. The estimated physical transient time is here $\tau_{\text {cond }}=0.32 \mathrm{~s}$ where $\delta$ is taken as the solid domain size $L_{s}$ and $a_{s}=3.1 \times 10^{-6} \mathrm{~m}^{2} / \mathrm{s}$. This gives a dimensionless time $\tau_{\text {cond }} \cdot f_{\text {ext }}=31$ which is consistant with the non-accelerated solution shown in Fig. 3. The fluctuations amplitude in Fig. 3 is two orders of magnitude smaller than the one entering at $x=-L_{f}$. 


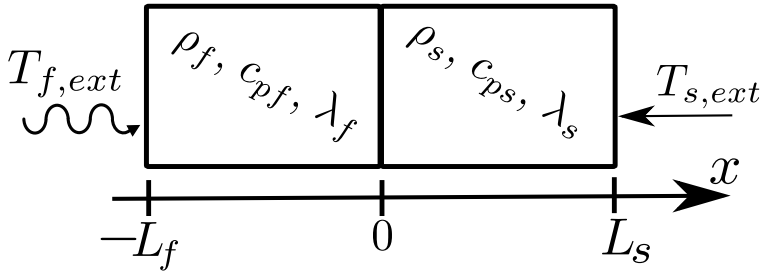

FIGURE 2. One-dimensional test case configuration. The length $L_{f}$ of the fluid part is $1 \mathrm{~mm}$ while the length $L_{s}$ of the solid part is $1 \mathrm{~mm}$.

This effect can be attributed to the value of the thermal activity ratio $K\left(=4.7810^{-3}\right.$ in this case $)$, defined as:

$$
K=\sqrt{\frac{\rho_{f} c_{p f} \lambda_{f}}{\rho_{s} c_{p s} \lambda_{s}}} .
$$

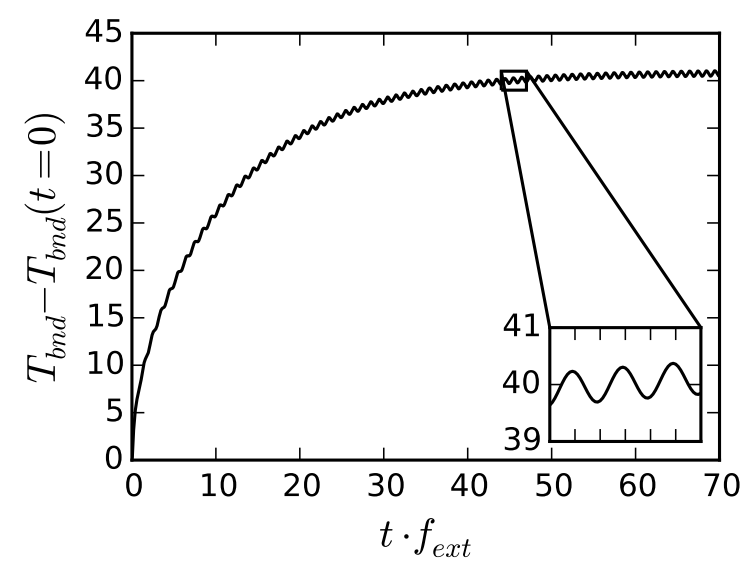

FIGURE 3. Temporal evolution of the boundary temperature for $f_{\text {ext }}=100 \mathrm{~Hz}$.

Results for the described superposition approach are compared with a reference solution (sufficiently refined to neglect discretization errors) without any artificial acceleration (here, affordable in 1D) and with a standard Neumann-Dirichlet coupling method accelerated by the desynchronization method $[5,9,17]$ with the factor $\alpha=10$ and $\alpha=100$. For a better assessment of the methods, the boundary temperature evolution is split into two components. The first one is the evolution of the pseudomean temperature $\widehat{T}_{\text {bnd }}$ defined as in Eq. 5 at the fluid/solid interface. Tracking its evolution allows a better vision of the convergence speed of the calculated mean temperature field towards its steady value. The second component studied is the fluctuating part of the boundary temperature, generated by the oscillating outer boundary condition.

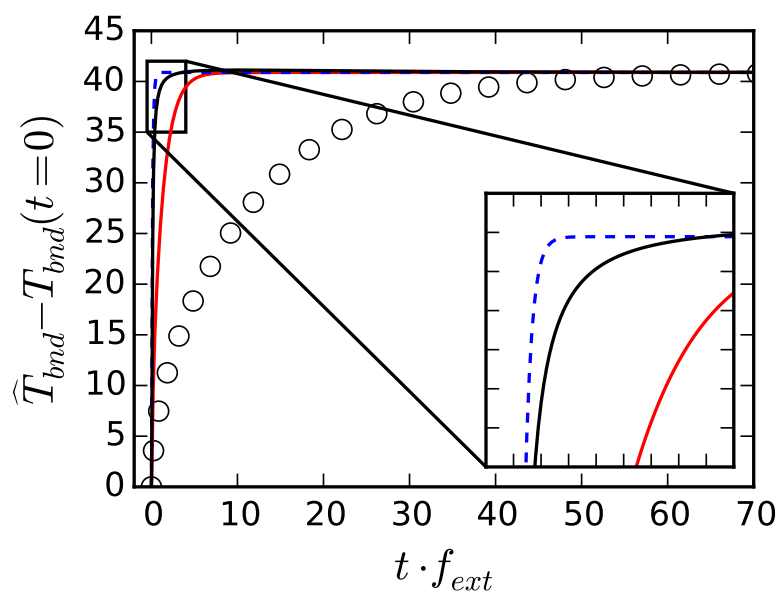

FIGURE 4. Temporal evolution of the mean component of the boundary temperature. Circles: Non-accelerated reference case. Red plain line: Neumann-Dirichlet coupling approach with a desynchronization factor $\alpha=10$. Blue dashed line: Neumann-Dirichlet coupling approach with a desynchronization factor $\alpha=100$. Black plain line: The HCND coupling approach with the derived acceleration method based on the superposition of mean and fluctuating states. Case conditions: $f_{\text {ext }}=100$ $\mathrm{Hz}$ and prescribed tolerance $\eta=5 \%$ for the HCND approach.

The mean component of the boundary temperature is plotted in Fig. 4. The reference solution shows that the transient heating determined by the solid time scale corresponds approximatively to fifty periods of the chosen value of $f_{\text {ext }}$. Desynchronizing the solid physical time in respect to the one seen in the fluid enables to reach the steady plateau much faster. With different values of the desynchronization factor $\alpha$, convergence can be made arbitrarily short although one must still pay attention to the numerical stability of the coupling method [5]. The proposed method also enables to quickly reach the steady value of the boundary temperature, much sooner than in the reference solution. The plateau reached by the symbols on the right of Fig. 4 is the steady mean temperature to retrieve. Both acceleration predicts accurately this mean value after the accelerated transient.

Figure 5 shows the unsteady part of the interface temperature. According to the reference solution, the imposed fluctuations yield an amplitude of approximatively 1 Kelvin at the interface. A known weakness of the desynchronization method is seen here: The heat flux fluctuations received by the solid wall 
have a frequency which is scaled by $\alpha$ and hence a response amplitude which is multiplied by $\sqrt{\alpha}$ [17]. On the other hand, the proposed superposition approach of the mean and unsteady parts provides an excellent agreement after an initial transient time. These first five or six signal periods have indeed been influenced by the pseudo-mean temperature value which has not converged yet. However, once the permanent regime is reached, the predicted fluctuations are accurate, which is the purpose of the acceleration method.
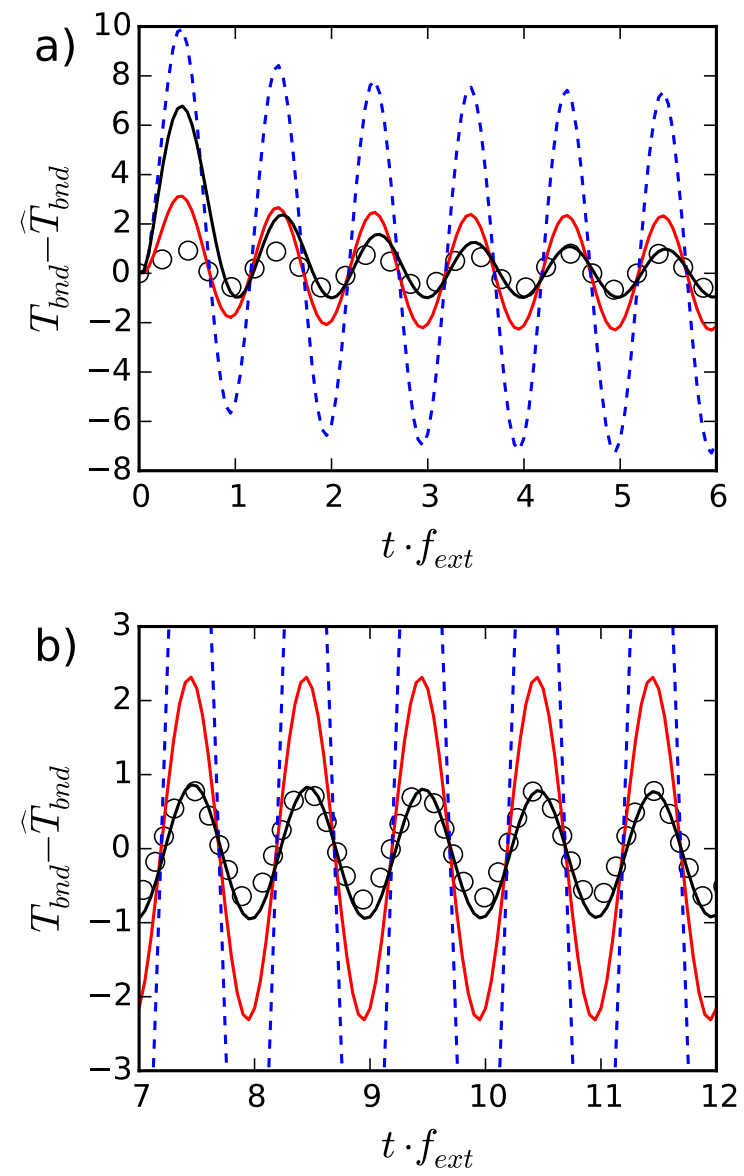

FIGURE 5. Temporal evolution of the unsteady component of the boundary temperature. (a) $t \cdot f_{\text {ext }}$ between 0 and 6 . (b) Zoom in the permanent regime for $t \cdot f_{\text {ext }}$ between 7 and 12 . Same line and symbol conventions as in Fig. 4.

The combination of the proposed acceleration method and HCND coupling method successfully predicts the steady wall temperature and heat flux in the permanent regime without perturbing their unsteady contributions and while automatically de- termining the coupling time step. This approach, validated in 1D cases, is applied to the study of flame-wall interaction in the next section.

\section{FLAME-WALL INTERACTION CONFIGURATION: STEADY REGIME}

The accelerated Hybrid-Cell Neumann-Dirichlet coupling method is applied to a three-dimensional conjugate heat transfer problem of a wall-impinging flame. Given the moderate Reynolds number, the flow is quite stable with a marginal unsteadiness. This section's objective is to predict the steady wall temperature with the enhanced HCND method. In spite of the studied flow being laminar and of the configuration being axisymmetric, the general objective is to deal with complex geometries and turbulent flows in LES with massively parallel codes. That is why the retained set of solvers, detailed below, is identical to the one used for such complex cases. This enables to assess the developed multiphysics simulation framework in a simpler and well-controlled configuration. In the next section, the flow is pulsated to enhance the flame unsteadiness and highlight all the benefits of the accelerated HCND coupling approach.

\section{Description of the experimental setup}

The studied experimental setup is shown in Fig. 6. It consists of a jet flame impinging a solid disc which is cooled on its other side, which was investigated by Singh et al. [19]. The flow is composed of a central jet (methane-air mixture with an equivalence ratio of 0.83 ) surrounded by a co-flow of pure nitrogen. The jet Reynolds number based on the injector diameter at the exit plane is 2,500 and the co-flow bulk velocity is $20 \%$ of the jet bulk inlet velocity. Given the low Reynolds number, the flow is laminar at the jet exit plane flow and features some small unsteadiness, generated by the shear layer between the premix jet and nitrogen coflow.

Available measurements [19] include the disc's mean surface temperature using Phosphor thermometry. Temperature and $C O$ concentrations in the gas phase have also been measured using Coherent Stokes Raman Spectroscopy (CARS) and LaserInduced Fluorescence (LIF), respectively. The same experimental configuration has been studied experimentally and numerically under different operating conditions and using a perforated plate to enhance turbulence: An equivalence ratio of 1.0 and a jet Reynolds number ranging from 5,000 to 50,000 [21], as well as a study of the unsteady evolution of the flame from ignition to stabilization against the wall has also been investigated experimentally [22] with a Reynolds number of 5,000.

\section{Physical modeling and numerical setup}

The multiphysics simulation relies on separate parallel solvers to describe each physics that communicate with each 


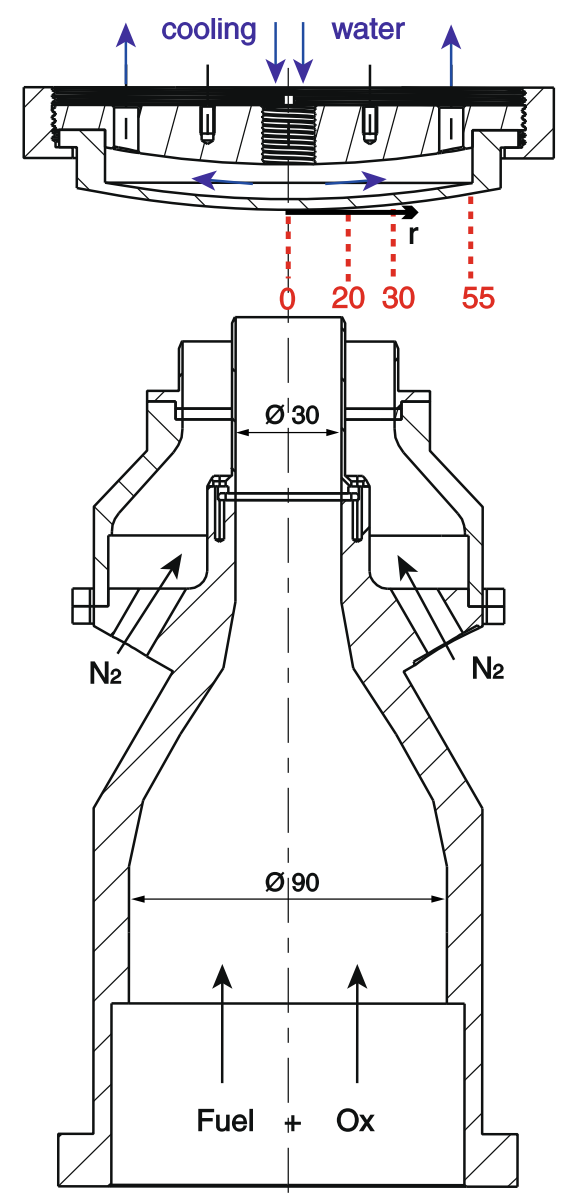

FIGURE 6. Experimental setup studied by Singh et al. [19]. The disc is located at a distance equal to one diameter from the injector.

other through a coupling procedure. One solver deals with the reactive flow, while two instances (steady and unsteady contributions) of the heat conduction solver determines the temperature field within the solid domain.

Reactive flow-solver Accounting for the detailed geometry shown in Fig. 6, the mesh is made of 57 million tetrahedra. The main zone of interest is located in the 12-mm-long region close to the disc surface where the cell size is about 0.25 $\mathrm{mm}$, which is enough to capture the gradient of the experimental temperature profile at the wall (see Fig. 11).

Simulations are carried out with the massively parallel low Mach-number DNS/LES solver YALES2 [23]. It is a finitevolume vertex-centered code, using fourth order space discretization and fourth-order Runge-Kutta like scheme. This 3D unsteady solver is considered for the investigated laminar flame to assess the methodology in a simple case while retaining the same numerical choices as for more practical cases of turbulent flames. Combustion of methane is described by the Coffee mechanism [24] that involves 14 species and 38 reactions. In order to handle the stiffness induced by chemistry, a splitting method is used and chemical source terms are integrated with the RADAU5 solver [25].

Given the moderate Reynolds number of the jet, the flow is resolved enough so that no LES model is needed. Nonetheless, capturing the flame thickness on the mesh remains too expansive. The flame thickening technique $[26,27]$ is then considered instead: By artificially modifying the diffusive fluxes $\mathscr{J}_{k}$ and the reaction rates $\dot{\omega}_{k}$ with a thickening factor $F$ as $\mathscr{J}_{k} \rightarrow F \mathscr{J}_{k}$ and $\dot{\omega}_{k} \rightarrow \dot{\omega}_{k} / F$, the flame is broadened but maintains its intrinsic laminar burning speed. A dynamic thickening is here used in order to limit the thickening to the reaction zone only, and prevent unwished modifications of the wall fluxes [28]. For the flame-wall interaction studied here, it is indeed mandatory not to modify the heat fluxes at the walls. To do so, a flame sensor based on the definition of a progress variable $Y_{c}$ and its reaction rate $\dot{\omega}_{Y_{C}}$ is used, with $Y_{c}=Y_{C O}+Y_{C_{2}}+Y_{H_{2} O}$ and $\dot{\omega}_{Y_{c}}=\dot{\omega}_{C O}+\dot{\omega}_{\mathrm{CO}_{2}}+\dot{\omega}_{\mathrm{H}_{2} \mathrm{O}}$. The flame sensor is then defined as:

$$
S_{F}=\max \left(1, \frac{A_{F} \dot{\omega}_{Y_{c}}}{\dot{\omega}_{\text {threshold }}}\right)
$$

The thickening amplitude $A_{F}$ depends on the local mesh size so that the flame could be correctly solved as in [29]. Finally, the local dynamic thickening factor can be computed at each point of the mesh as $F=1+\left(A_{F}-1\right) S_{F}$. In the zone of interest where the flame is stabilized, the thickening factor is approximatively 6 .

Solid solver The unsteady conjugate heat transfer methodology described in this study requires an unsteady heat transfer solver but also a steady heat solver when the acceleration method is used. Both are instances of the YALES2 framework: the data structure and discretization schemes are identical to the flow solver. Hence, the unsteady heat transfer solver is a finite volume solver using fourth order schemes for space discretization and explicit time integration. For the steady solver, the steady heat equation is solved using a deflated conjugate gradient method [30]. The method is identical to the one used to solve the Poisson equation on pressure in the low Mach-number solver.

The mean and fluctuating temperature in the disc heated by the flame on one side and cooled on the other side are determined by Eqs. 2-3 which are solved by the combination of both heat solvers. On the cooled side, the temperature is imposed on the experimentally measured profile, i.e., between $330 \mathrm{~K}$ in the center and $340 \mathrm{~K}$ at the disc's extremity. The mesh of the solid part consists of approximately 8 million tetrahedral $0.25-\mathrm{mm}$-wide cells. The solid cell size is similar to the fluid cell size close to 
the wall. A zoom of the zone of interest, showing both gaseousflow and solid meshes, is presented in Fig. 7. The quartz constant properties (taken for $T=400 \mathrm{~K}$, the intermediate temperature between the cooled side and the flame side) are the following : $c_{p_{s}}=954 \mathrm{~J} / \mathrm{kg} / \mathrm{K}, \rho_{s}=2200 \mathrm{~kg} / \mathrm{m}^{3}$ and $\lambda_{s}=1.732 \mathrm{~W} / \mathrm{m} / \mathrm{K}$.
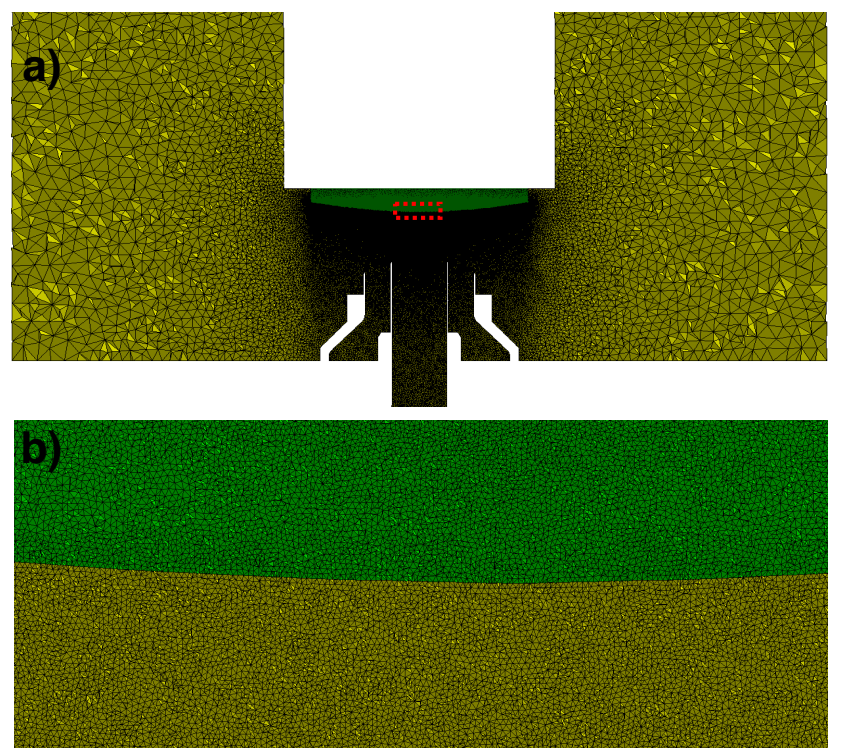

FIGURE 7. Cuts of fluid (yellow) and solid (green) meshes. (a) Injection burner, coflow and solid disc. (b) Zoom (red square in top figure) around the interface.

Coupling procedure The coupling communications' scheme between the different parts is presented in Fig. 8. While Fig. 8 highlights the different fields to compute and how they interact, there is in fact no separate solver for the boundary temperature. For the sake of practical and efficient implementation, the boundary temperature solver is integrated inside each of the two unsteady solvers (gaseous flow and solid heat transfer). Hence, only integrated heat fluxes are actually exchanged between unsteady solvers and there is no noticeable computational overhead between the HCND and the standard Neumann-Dirichlet coupling approaches [18] .

All exchanged data between solvers are communicated with the coupling library OpenPALM [31] which is developed for massively parallel coupled simulations. Flexibility in the coupling settings and efficiency are gained thanks to OpenPALM which provides generic coupling subroutines, and handles the efficient and scalable dispatching of the sent information to the correct recipient.

The coupled simulation uses 984 cores divided as follows:

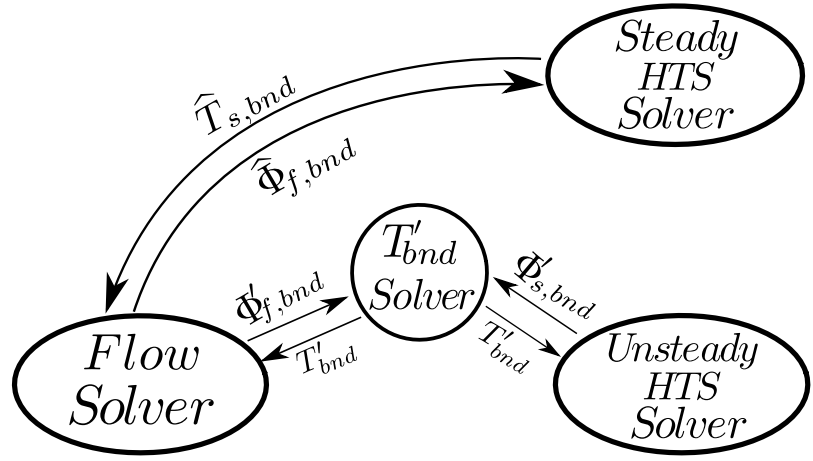

FIGURE 8. Scheme of the NCND method for coupled unsteady conjugate heat transfer when using acceleration of the physical transient heating or cooling.

1 core for the OpenPALM coupler, 23 for the solid heat transfer solver and the remaining 960 cores were used by the reactive flow solver. The initial temperature field in the solid disc is set to $300 \mathrm{~K}$. For the flow solver, the initial fields of velocity, temperature and species are obtained from a reactive non-coupled simulation with a $300 \mathrm{~K}$ disc temperature. The characteristic thermal time scale of the quartz disc is of about 30 seconds, thus leading to a computational cost of approximately 4 million CPU hours to physically reach the permanent state of the heated disc. As explained previously, this cost is prohibitive and outlines the need for an artificial acceleration of the solid domain transient heating. Thanks to such an acceleration, the complete cost of one coupled simulation is of about $100000 \mathrm{cpu}$ hours on a BullX cluster with Intel Xeon processors (E5-2690v3).

For comparison with the derived acceleration for the HCND method, an additional coupled simulation is carried out with a standard Neumann-Dirichlet coupling approach with a desynchronization factor $\alpha=1000$. The HCND method automatically adapts the coupling step size, here with a prescribed tolerance $\eta=5 \%$. The resulting variable coupling period during the transient heating of the disc is shown in Fig. 13. Since the second coupling approach, the desynchronized Neumann-Dirichlet method, does not determine the coupling period, it is fixed to 800 diffusive flow time steps (the approximative value obtained in the steady regime of Fig. 13).

\section{Reactive flow results}

A field cut of the obtained mean temperature is shown in Fig. 9. The flame (A) is seen to stabilize about $8 \mathrm{~mm}$ from the wall. On the sides, the large region (B) of intermediate temperature around $1000 \mathrm{~K}$ is due to dilution of burnt gases with the nitrogen coflow.

When carrying out flame thickening while studying wall heat transfer, particular attention must be paid to the flame sensor 


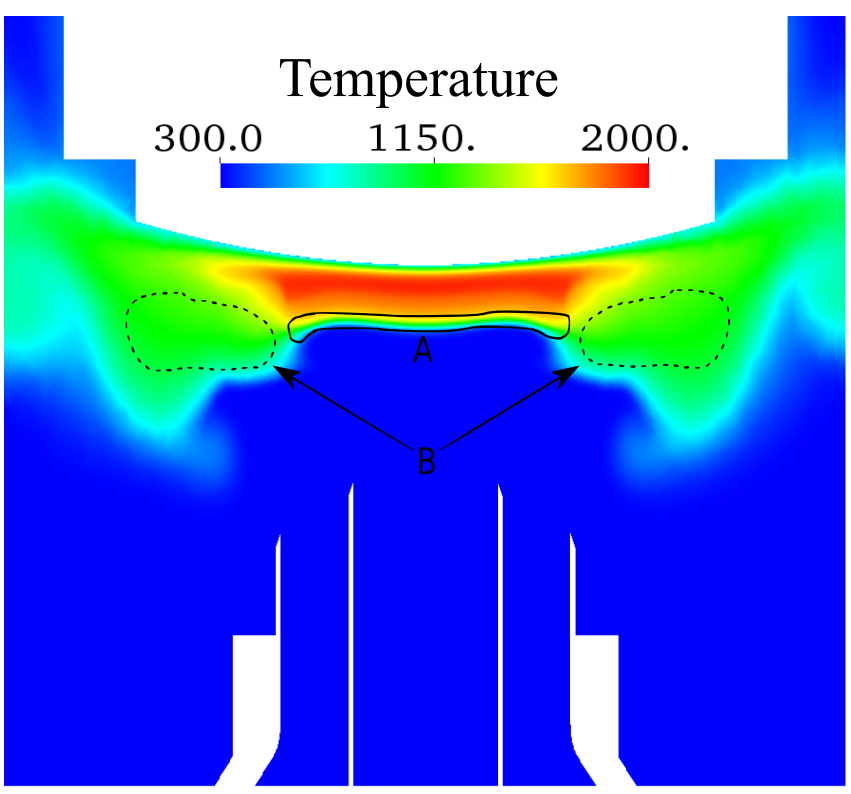

FIGURE 9. Field cut of the gas mean temperature. A: Reaction zone delimited by the iso-line $\dot{\omega}_{Y_{c}}=0.1 \dot{\omega}_{Y_{c}}^{\max }$. B: Dilution zones where the hot gases encounter the coflow of $N_{2}$.

that locally activates the thickening procedure. Indeed, by artificially altering the diffusion coefficients to increase the flame thickness, flame thickening modifies the temperature gradient and hence the diffusive heat flux. That is why, an accurate conductive wall heat flux requires the sensor not to be activated at the walls. Such a requirement becomes impractical when the flame actually impacts and interacts with the wall. In the studied configuration, while close to the wall, the flame remains $8 \mathrm{~mm}$ millimeter away from it. Figure 10 shows the mean value of the flame sensor along the jet axis with the profiles of temperature and reaction rate. The thickened zone is about $5-\mathrm{mm}$ wide and centered around the flame position. The thickened zone englobes the reaction zone as well as the rapid temperature increase due to the chemical reactions. For the burnt gases cooled in the vicinity of the wall, the sensor is null ensuring that the wall heat flux is not perturbed.

Experimental and numerical profiles of temperature along the central axis are plotted in Fig. 11. The agreement between both profiles is very good as for the wall heat flux on the disc at the radial position $r=0$ : the experimental value is $69.8 \mathrm{~kW} / \mathrm{m}^{2}$ while it is evaluated to $70.5 \mathrm{~kW} / \mathrm{m}^{2}$ in the numerical simulation. The value is slightly overestimated because the computed temperature value is below the measured value, as seen later in Fig. 14.

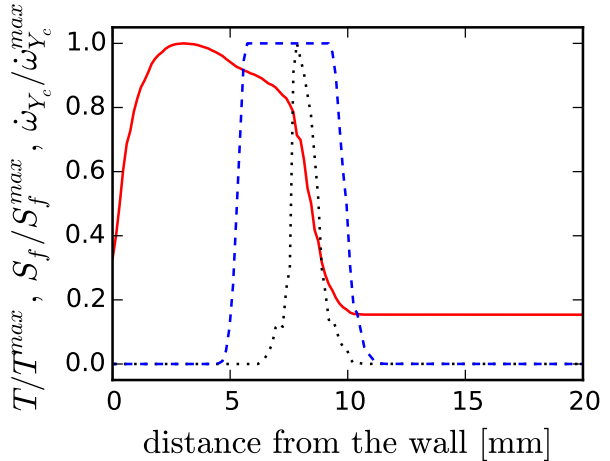

FIGURE 10. Profiles of the normalized temperature (red plain line), flame sensor $S_{f}$ (blue dashed line) and progress variable source term $\dot{\omega}_{Y_{c}}$ (black dotted line) along the centerline axis.

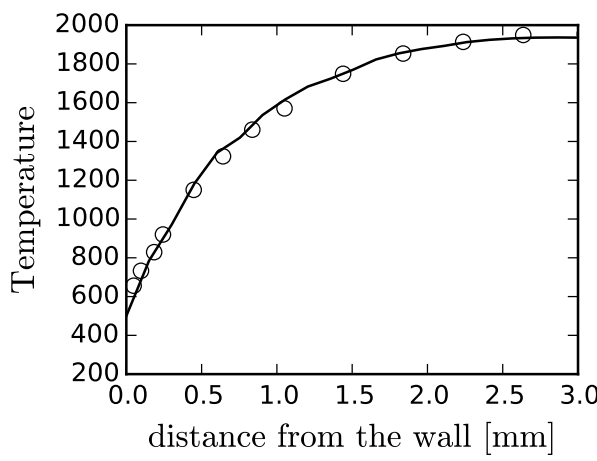

FIGURE 11. Comparison of the experimental (symbols [19]) and numerical (plain line) mean temperature in the near wall region along the centerline axis

\section{Wall temperature results}

Figure 12 shows the temporal evolution of the boundary temperature at the disc center $(r=0)$. The reference unaccelerated solution is now unaffordable computationally with 3D unsteady simulations: artificial acceleration techniques are necessary to hasten the thermal transient heating. As shown in the $1 \mathrm{D}$ test case, the physical transient time can be estimated by the characteristic conductive time scale in the quartz $\tau_{\text {cond }}=\delta^{2} / a_{s}$ with the $\delta=3 \mathrm{~mm}$ is the quartz thickness and $a_{s}=8.2 \times 10^{-7} \mathrm{~m}^{2} / \mathrm{s}$. Hence, the computed order of magnitude of the transient heating of the quartz is $\tau_{\text {cond }}=10.9 \mathrm{~s}$. In both accelerated coupling methods (HCND with superposition and standard Neumann-Dirichlet with desinchronization) shown in Fig. 12 , the permanent regime is then achieved very fast (roughly 500 times faster) compared to a non-accelerated simulation. The fact that the duration of the accelerated transient is similar with 
both acceleration methods is fortuitous with the desynchronization factor $\alpha=1000$. The adapted coupling time step during the accelerated transient depicted in Fig. 13 is smaller during the initial $15 \mathrm{~ms}$ while the wall temperature increases and then reaches a plateau in the permanent regime.

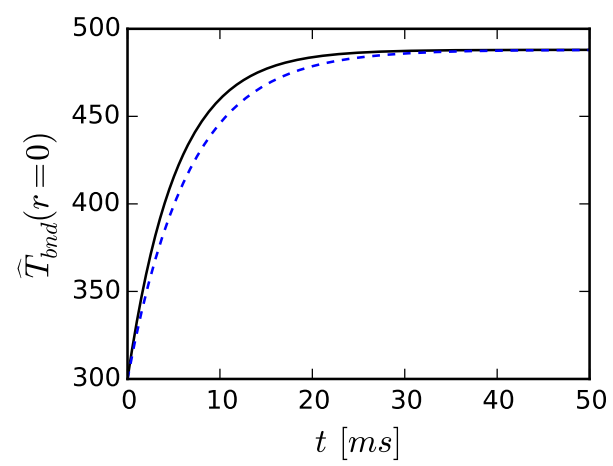

FIGURE 12. Temporal evolution of the computed mean contribution of the boundary temperature at the disc center for two acceleration methods. Black plain line: Hybrid-cell with a superposition based acceleration with $\eta=5 \%$. Blue dashed line: Neumann-Dirichlet coupling approach with a desynchronization factor $\alpha=1000$.

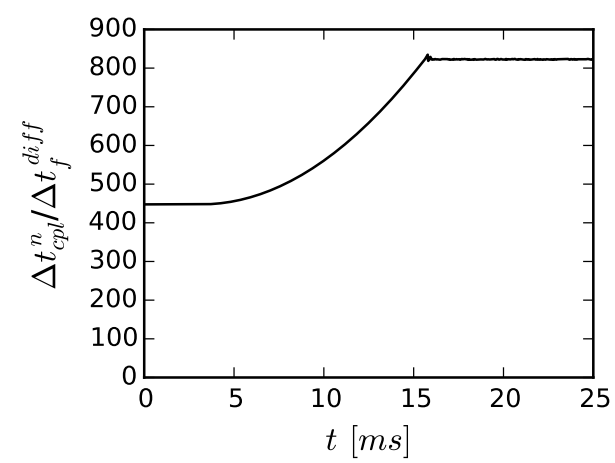

FIGURE 13. Temporal evolution of the coupling time step scaled by the flow solver limiting diffusive time step for the tolerance $\eta=5 \%$.

Radial profiles of temperature on the disc for both coupled simulations are compared with the experimental measurements in Fig. 14. The predicted mean temperature on the disc's hot surface is excellent for both methods with a local error below $1 \%$. This demonstrates that the derived accelerated HCND is able to perform as well as another standard approach to predict efficiently mean statistics in practical conjugate heat transfer problems. Additionally, the enhanced method introduces interesting features: self-adaptive coupling time step and no perturbations of fluctuations while acceleration is active. These two features are highlighted in the next section in a pulsated regime.

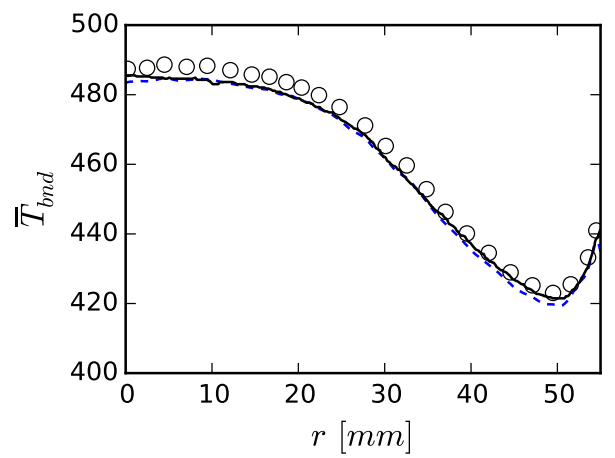

FIGURE 14. Comparison between experimental and numerical data for the mean boundary temperature as a function of the distance from the center of the disc. Circles: Experimental data [19]. Black plain line: Hybrid-cell interface model with a superposition-based acceleration, and $\eta=5 \%$. Blue dashed line: Neumann-Dirichlet coupling approach with a desynchronization factor $\alpha=1000$.

\section{FLAME-WALL INTERACTION CONFIGURATION: PUL- SATED REGIME}

In order to enhance the flow dynamics and induce a variable coupling time step during the permanent regime, the flame jet mass flowrate is pulsated in the coupled simulation. This purely numerical case enables to challenge the combination of acceleration and dynamic time step control of the HCND method in a truly unsteady conjugate heat transfer case.

\section{Description of the simulated conditions}

The mass flow rate of the premixed jet is pulsated with the following prescribed temporal function:

$$
\dot{m}(t)=\dot{m}_{0}+\dot{m}^{\prime} \sin \left(2 \pi f_{\text {ext }} t\right)
$$

where the amplitude of the fluctuating mass flow rate $\dot{m}^{\prime}$ is set to $15 \%$ of the nominal mass flow rate $\dot{m}_{0} . f_{\text {ext }}$ is taken equal to 40 $\mathrm{Hz}$. The physical modeling and numerical setup is identical to the previous case. 


\section{Flow unsteadiness and coupling time step control}

As shown in Fig. 15, the induced pulsations of velocity create Kelvin-Helmholtz instabilities inside the jet-coflow shear layer. The generated vortices are then conveyed downstream where they perturb the flame front (see Fig. 15). The resulting

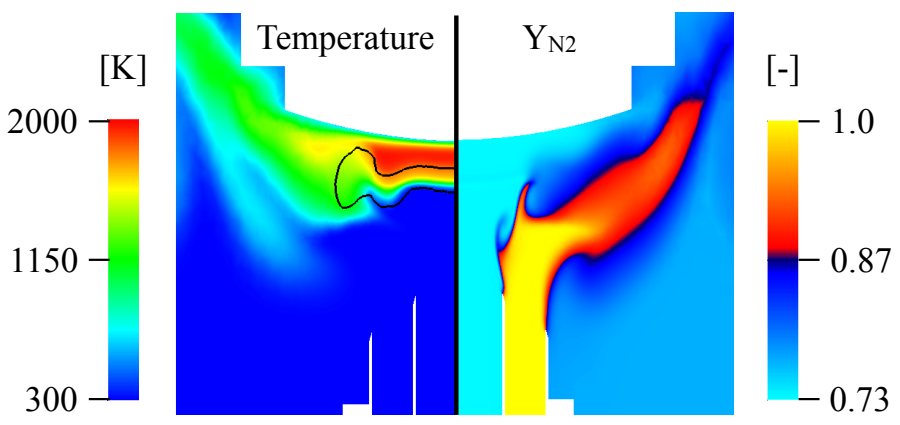

FIGURE 15. Instantaneous colored map of temperature (left) and $\mathrm{N}_{2}$ mass fraction (right) in the pulsated configuration. Left: the black contour indicates the flame position as in Fig. 9

changes in the disc temperature variations are shown in Fig. 16 (top) on the centreline $r=0$. Temperature fluctuations are lower than one Kelvin. The frequency of the oscillations in the wall temperature and conductive flux (not shown) is identical to the one prescribed in the inlet condition. Variations in the wall conductive flux at $r=0$ (not shown) are similar to the one seen for the disc temperature while the relative amplitude is larger: 0.15 $\%$ for the disc temperature and $0.89 \%$ for the heat flux.

Figure 16 (bottom) also shows the corresponding evolution of the coupling time step since the beginning of the pulsated case simulation. First, the coupling step remains identical to the steady configuration because vortices have not reached the wall yet. The fact that the duration of this first phase is almost one period of the pulsated inlet is due to the chosen frequency of $40 \mathrm{~Hz}$ being close to the actual convection time $D_{i n j} / U_{i n j}^{b u l k}$. Once the coherent structures perturb the flame and modify the wall heat flux, the coupling step size decreases instantly and stabilizes at a lower value than the initial one. Once the permanent regime is reached (observed for $t \cdot f_{\text {ext }} \geq 5$ approximatively), an alternation of peak values is seen to occur twice during a period of the mass flow rate's oscillations. This result is similar to the one observed in [18] while characterizing the HCND controller in unsteady 1D cases.

\section{Root-mean-square statistics at the disc surface}

The same pulsated case has also been simulated using a Neumann-Dirichlet coupling approach with desynchronization. As explained previously, this method is expected to amplify the
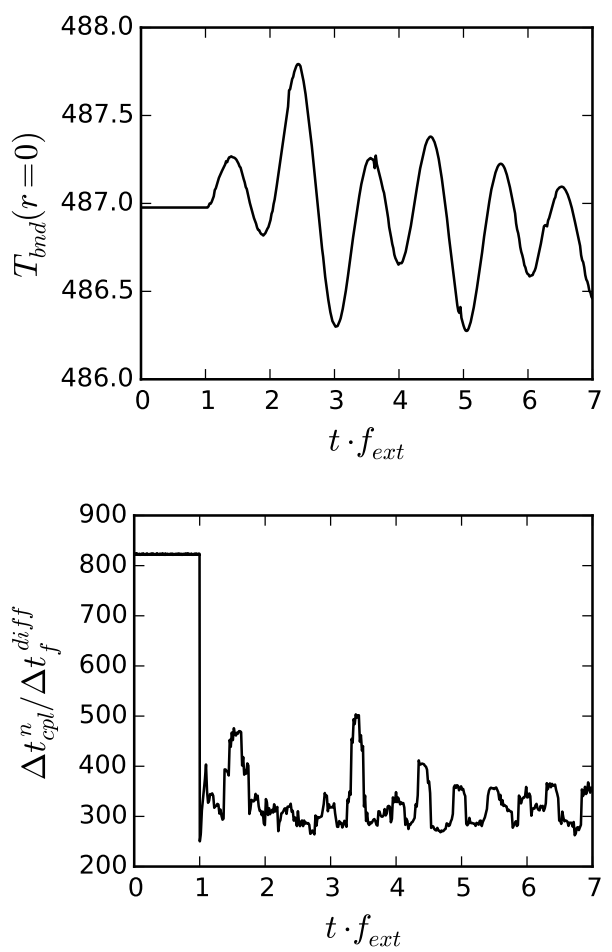

FIGURE 16. Temporal evolution of the boundary temperature (top) at the center position of the disc and of coupling time step (bottom) scaled by the flow solver limiting diffusive time step as determined by the accelerated HCND method.

wall fluctuations. One could think of a possible fix for this issue by removing the desynchronization procedure once the permanent has been reached. However, there is a disadvantage in this stopped desynchronization than can be foreseen: While using desynchronization, the instantaneous thermal fields in the solid are the sum of the correct steady mean field and a wrongly amplified fluctuating part. When stopping the desynchronization to capture the correct unsteadiness, the duration of the transition from the wrong level of fluctuations due the correct one is at least determined by the physical conduction time scale in the solid. This duration is too long to capture since this was the purpose of using an acceleration technique in the first place. This is why a permanent desynchronization acceleration has been considered for the standard Neumann-Dirichlet coupling in the previous comparisons. The same choice is made in the following section.

In this studied pulsated configuration, the mean fields for both acceleration techniques are again very similar (not shown). Radial profiles of the root-mean-square of wall temperature on the disc for both acceleration techniques is shown in Fig. 17 (top). It is seen that the temperature RMS at the disc 

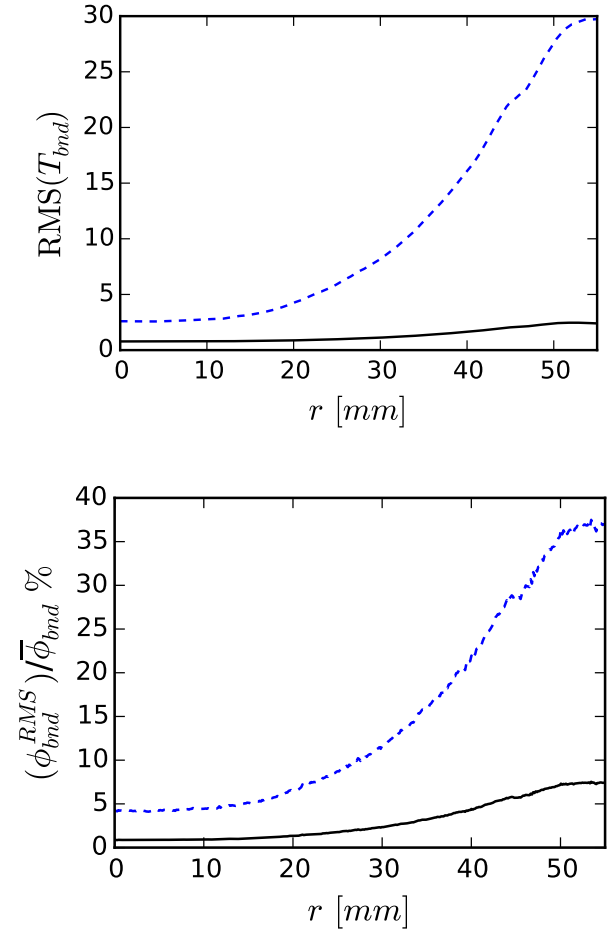

FIGURE 17. Radial profiles of the disc temperature RMS (top) and relative wall heat flux RMS (bottom) computed by the accelerated HCND approach (black plain line) and by the accelerated NeumannDirichlet coupling with a desynchronization factor $\alpha=1000$ (blue dashed line).

center is in fact smaller than the one observed close to the disc's edges for both accelerated methods. A similar trend is shown for the wall heat flux in Fig. 17 (bottom) which is characterized by larger relative variations.

The temperature root-mean-square profile of the desynchronized Neumann-Dirichlet method is one-order-of-magnitude larger than the accelerated hybrid cell approach. Similarly, the wall heat flux fluctuations are also overestimated. The desynchronization acceleration is known to amplify the computed fluctuations. Regarding the boundary temperature RMS, the theoretical amplification value of $\alpha^{1 / 2} \approx 32$ is valid for skin effect in a semi-infinite domain. This theoretical value overestimates the actual RMS amplification because the modified frequency induced by the desynchronization and the actual disc thickness are not compatible with a semi-infinite domain assumption. As highlighted in the 1D test cases, the accelerated hybrid cell approach does not perturb the level of fluctuations seen at the disc's surface contrary to the desynchronization approach. That is why the observed fluctuations are much lower for the accelerate HCND method. This present numerical test case lacks a true reference solution or experimental data to validate the RMS fields predicted by the developed method. Future applications will consider more practical cases with larger unsteady heat loads and experimental data for validation.

The numerically investigated pulsated configuration shows that the developed coupling framework (HCND with automatic determination of the coupling time step and transient acceleration) is able to deal with abrupt modifications of flow conditions and can adapt the coupling time step to the dynamics of each particular configuration.

\section{CONCLUSION}

The Hybrid-Cell Neumann-Dirichlet method for coupled unsteady conjugate heat transfer using large-eddy or direct numerical simulations has been used to compute a wall-impinging flame configuration. This coupling approach is conservative and allows an automatic determination of the coupling time step to adapt the temporal resolution to the studied configuration. In order to determine permanent regime statistics, an acceleration method consistent with this method has been derived in order to artificially accelerate the transient slow heat conduction in the solid without impacting the studied fluctuations of wall temperature and heat flux.

The enhanced accelerated HCND method is then applied to a quasi-steady wall-impinging flame configuration after modeling the reactive flow properly. In particular, the Coffee mechanism is considered to describe the methane oxidation. While the flow is fully resolved, the thickened flame for LES (TFLES) model is used to capture the thin flame on the mesh. Results of the coupled simulation show that the temperature profile on the flame side is correctly predicted. Thanks to the acceleration of the method, computing the radial profile of the wall temperature becomes affordable. The agreement with the experimental data and with another coupling method (the standard NeumannDirichlet approach) is very good.

In the pulsated case, and as expected from theory and 1D test cases, the desynchronization technique is shown to yield a larger magnitude of fluctuations at the wall than the accelerated HCND method which was developed not to perturb such unsteadiness. Validation of the HCND results remains however to be validated in more practical cases with larger unsteady heat loads and in comparison with experimental data. This is quite promising for applications such as thermal fatigue where the dynamics of unsteady conjugate heat transfer is critical. Finally, thanks to the HCND method, the coupling time step adapts automatically to the dynamics of each particular configuration, highlighting the interest of having an on-the-fly control of the coupling period.

Regarding future perspectives of the flame simulation, let us outline the need to address numerically a stronger flame-wall interaction with an impacted flame reaction zone, which is of high importance in ICEs. Since the classical TFLES model is not valid 
in this case due to the wrongly amplified wall heat flux, such configurations is also a real combustion modelling challenge.

\section{Acknowledgment}

This work was supported by the Air Liquide, CentraleSupélec and CNRS Chair on oxycombustion and heat transfer for energy and environment and by the OXYTEC project, grant ANR-12-CHIN-0001 of the French Agence Nationale de la Recherche. This work was granted access to the HPC resources of IDRIS under the allocations 2014-x20142b0164 and 2015x20142b0164 made by GENCI. Vincent Moureau and Ghislain Lartigue from CORIA, and the SUCCESS scientific group are acknowledged for providing the YALES2 code. Pr. Andreas Dreizler and his collaborators are acknowledged for providing the geometries and useful information concerning the experimental setup.

\section{REFERENCES}

[1] B. Facchini, A. Magi, A. S. Del Greco, Conjugate heat transfer simulation of a radially cooled gas turbine vane, in: ASME Turbo Expo 2004: Power for Land, Sea, and Air, American Society of Mechanical Engineers, 2004, pp. 951-961.

[2] W. D. York, J. H. Leylek, Three-dimensional conjugate heat transfer simulation of an internally-cooled gas turbine vane, in: ASME Turbo Expo 2003, collocated with the 2003 International Joint Power Generation Conference, American Society of Mechanical Engineers, 2003, pp. 351-360.

[3] A. Andreini, A. Bonini, R. Da Soghe, B. Facchini, A. Ciani, L. Innocenti, Conjugate heat transfer calculations on gt rotor blade for industrial applications: Part ii-improvement of external flow modeling, in: ASME Turbo Expo 2012: Turbine Technical Conference and Exposition, American Society of Mechanical Engineers, 2012, pp. 681-692.

[4] L.-Z. Zhang, C.-H. Liang, L.-X. Pei, Conjugate heat and mass transfer in membrane-formed channels in all entry regions, International Journal of Heat and Mass Transfer 53 (5-6) (2010) 815 - 824.

[5] F. Duchaine, A. Corpron, L. Pons, V. Moureau, F. Nicoud, T. Poinsot, Development and assessment of a coupled strategy for conjugate heat transfer with large eddy simulation: Application to a cooled turbine blade, International Journal of Heat and Fluid Flow 30 (6) (2009) 1129 - 1141.

[6] S. Jauré, F. Duchaine, G. Staffelbach, L. Gicquel, Massively parallel conjugate heat transfer methods relying on large eddy simulation applied to an aeronautical combustor, Computational Science and Discovery 6 (1) (2013) Article 015008.

[7] Y. Li, S.-C. Kong, Coupling conjugate heat transfer with in-cylinder combustion modeling for engine simulation, In- ternational Journal of Heat and Mass Transfer 54 (11-12) (2011) 2467 - 2478.

[8] E. Radenac, J. Gressier, P. Millan, Methodology of numerical coupling for transient conjugate heat transfer, Computers \& Fluids 100 (2014) 95 - 107.

[9] S. Berger, S. Richard, F. Duchaine, G. Staffelbach, L. Gicquel, On the sensitivity of a helicopter combustor wall temperature to convective and radiative thermal loads, Applied Thermal Engineering 103 (2016) 1450-1459.

[10] R. Mari, B. Cuenot, J.-P. Rocchi, L. Selle, F. Duchaine, Effect of pressure on hydrogen/oxygen coupled flame-wall interaction, Combustion and Flame 168 (2016) 409-419.

[11] L. Y. M. Gicquel, G. Staffelbach, T. Poinsot, Large eddy simulations of gaseous flames in gas turbine combustion chambers, Progress in Energy and Combustion Science 38 (6) (2012) 782-817.

[12] M. B. Giles, Stability analysis of numerical interface conditions in fluid-structure thermal analysis, Iternational Journal for Numerical Methods in Fluids 25 (4) (1997) 421436.

[13] L. He, M. Oldfield, Unsteady conjugate heat transfer modelling, ASME Journal of Turbomachinery 133 (3) (2011) 031022.

[14] L. He, Fourier spectral modelling for multi-scale aerothermal analysis, International Journal of Computational Fluid Dynamics 27 (2) (2013) 118-129.

[15] M. P. Errera, F. Duchaine, Comparative study of coupling coefficients in dirichlet-robin procedure for fluid-structure aerothermal simulations, Journal of Computational Physics 312 (2016) 218-234.

[16] M.-P. Errera, S. Chemin, Optimal solutions of numerical interface conditions in fluid-structure thermal analysis, Journal of Computational Physics 245 (2013) 431 - 455.

[17] S. Jaure, F. Duchaine, G. Staffelbach, L. Y. M. Gicquel, Massively parallel conjugate heat transfer methods relying on large eddy simulation applied to an aeronautical combustor, Computational Science and Discovery 6 (1) (2013) 015008.

[18] C. Koren, R. Vicquelin, O. Gicquel, Self-adaptive coupling frequency for unsteady coupled conjugate heat transfer simulations, International Journal of Thermal Science (Submitted).

[19] A. Singh, M. Mann, T. Kissel, J. Brübach, A. Dreizler, Simultaneous measurements of temperature and co concentration in stagnation stabilized flames, Flow Turbulence and Combustion 90 (4) (2013) 723-739.

[20] G. Söderlind, Digital filters in adaptive time-stepping, ACM Transactions on Mathematical Software (TOMS) 29 (1) (2003) 1-26.

[21] P. Pantangi, A. Sadiki, J. Janicka, M. Mann, A. Dreizler, Les of premixed methane flame impinging on the wall using non-adiabatic flamelet generated manifold (FGM) ap- 
proach, Flow Turbulence and Combustion 92 (4) (2014) 805-836.

[22] M. Mann, C. Jainski, M. Euler, B. Böhm, A. Dreizler, Transient flame-wall interactions: Experimental analysis using spectroscopic temperature and CO concentration measurements, Combustion and Flame 161 (9) (2014) 2371 - 2386.

[23] V. Moureau, P. Domingo, L. Vervisch, Design of a massively parallel cfd code for complex geometries, Comptes Rendus Mecanique 339 (2-3) (2011) 141-148.

[24] T. Coffee, Kinetic mechanisms for premixed, laminar, steady-state hydrogen nitrous-oxide flames, Combustion and Flame 65 (1) (1986) 53-60.

[25] E. Hairer, G. Wanner, Stiff differential equations solved by radau methods, Journal of Computational and Applied Mathematics 111 (1-2) (1999) 93-111.

[26] T. Butler, P. O'Rourke, A numerical method for two dimensional unsteady reacting flows, Symposium (International) on Combustion 16 (1) (1977) 1503 - 1515.

[27] O. Colin, F. Ducros, D. Veynante, T. Poinsot, A thickened flame model for large eddy simulations of turbulent premixed combustion, Physics of Fluids 12 (7) (2000) 18431863.

[28] J.-P. Légier, T. Poinsot, D. Veynante, Dynamically thickened flame les model for premixed and non-premixed turbulent combustion, in: Proc. of the summer program, Citeseer, 2000, pp. 157-168.

[29] G. Kuenne, A. Ketelheun, J. Janicka, Les modeling of premixed combustion using a thickened flame approach coupled with fgm tabulated chemistry, Combustion and Flame 158 (9) (2011) 1750 - 1767.

[30] M. Malandain, N. Maheu, V. Moureau, Optimization of the deflated conjugate gradient algorithm for the solving of elliptic equations on massively parallel machines, Journal of Computational Physics 238 (2013) 32 - 47.

[31] S. Buis, A. Piacentini, D. Déclat, Palm: a computational framework for assembling high-performance computing applications, Concurrency and Computation: Practice and Experience 18 (2) (2006) 231-245. 